\title{
EDITORIAL
}

\section{Advances in depression research: 2011}

\author{
Molecular Psychiatry (2011) 16, 686-687; doi:10.1038/ \\ $\mathrm{mp} .2011 .74$
}

This is a special issue of Molecular Psychiatry dedicated to advances in depression research. Major depressive disorder (MDD) is a substantial public health problem; it is the second cause of disability worldwide and it costs the US economy over 100 billion dollars annually. In the US, suicide, mostly a consequence of depression, is the 11th cause of death; it is the third cause of death in the 15-24 age group and the fourth cause of death in the 25-44 age group. ${ }^{1}$ In Australia depression represents the number 1 cause of non-fatal disease burden and the third fatal disease burden in Australian men. Antidepressants represent the second most widely sold class of drugs in America, the first one being analgesics. In 2008, more than 164 million prescriptions were written for antidepressants in the United States. Research in depression is therefore a public health priority. ${ }^{2} \mathrm{We}$ are very pleased that in this issue of Molecular Psychiatry, eight outstanding papers bring together the latest advances in this exciting and critically important area of investigation. These fall into three broad domains: (i) potential causes, (ii) pathways and mechanisms, and (iii) prevention and treatment.

\section{Potential causes}

The foremost question in depression research is causality. According to the paper by Demirkan et $a .^{3}$ from Rotterdam, The Netherlands, this will be hard to elucidate. They investigated the contribution of a large number of genes to complex traits by genome-wide profiling aimed at assessing the genetic risk for depression and anxiety in adult and elderly cohorts. Their findings suggest the presence of multiple genetic loci of very small effect that influence both depression and anxiety. This suggests that genes of major effect are not at play in anxiety and depression and therefore the elucidation of the genetics of depression will require very large samples for studies that have the power to identify with statistical significance a plethora of disease susceptibility genes of minor effect.

Looking at causality from a very specific perspective, Peñas-Lledó et al. ${ }^{4}$ from Badajoz, Spain showed in a brief report (letter to the editor) a high risk of lifetime history of suicide attempts among CYP2D6 ultrarapid metabolizers with eating disorders. This is important because the cytochrome P450 enzymes, particularly CYP2D6, have been traditionally viewed only through their key role in pharmacokinetics:
CYP2D6 metabolizes $50 \%$ of the top 100 bestselling drugs in the US. The conceptually novel work from Peñas-Lledó et al. shows that CYP2D6 may have a clinically important role in intrinsic functions of the human brain, such as regulation of mood and suicidality. This work opens a new door for investigating the mechanisms for this interesting and clinically relevant association.

\section{Pathways and mechanisms}

These are illustrated by original articles as well as by a feature review by van Donkelaar et al., ${ }^{5}$ from Maastricht, The Netherlands, in which the authors question whether the mechanisms of the effects observed during experiments of acute tryptophan depletion are indeed solely based on serotonin. Taking into account their rigorous review of existing evidence, these authors suggest that caution is required when interpreting the effects of acute tryptophan depletion in terms of a selective serotonergic effect.

Over 25 years of research have pointed out to a key role of corticotropin-releasing factor (CRF) as a biological pathway for major depression. While hypothalamic CRF activates the hypothalamic pituitary adrenal axis, CRH in extra-hypothalamic sites, such as the amygdala, has key roles in behavior. Regev et $a .^{6}{ }^{6}$ from Rehovot, Israel, showed that prolonged and site-specific overexpression (OE) of CRF reveals differential roles for extended amygdala nuclei in emotional regulation. Their elegant experiments, which used lentivirus constructs, demonstrated that prolonged CRF overexpression at the central nucleus of the amygdala attenuated stress-induced anxietylike behaviors, whereas prolonged CRF OE in the dorsolateral subdivision of the bed nucleus of the stria terminalis increased depressive-like behaviors, without affecting anxiety levels. These results provide evidence for differential roles for CRF expressed by distinct loci of the extended amygdala in mediating stress-induced emotional behaviors. This line of work raises caution at translational drug development of CRF antagonists, which have been long sought as conceptually novel antidepressants. Such drugs might have divergent effects depending on the predominant site of action of particular compounds.

What is happening inside the brain of patients suffering from major depression? Two of the papers published in this issue address this fundamental question at the neuropathological and functional levels. First, Shelton and colleagues ${ }^{7}$ from Nashville, USA studied postmortem brain tissue samples from 
Brodmann Area 10 (BA10) in the prefrontal cortex from psychotropic drug-free persons with a history of MDD and age, gender and postmortem intervalmatched normal controls. Using microarray they showed altered expression of genes involved in inflammation and apoptosis in the frontal cortex in major depression. Their exciting data indicate that postmortem brain tissue samples from BA10, a region that is involved in reward-related behavior, show evidence of local inflammatory, apoptotic and oxidative stress in MDD.

At the functional level, Hamilton et al., ${ }^{8}$ from Stanford, USA, investigated neural primacy in MDD, looking at multivariate Granger causality (GC) analysis of resting-state fMRI time-series data. They used multivariate GC analysis, a technique that estimates the extent to which preceding neural activity in one or more seed regions predicts subsequent activity in target brain regions, to analyze blood-oxygen-leveldependent data collected during eyes-closed rest state from depressed and never-depressed persons, and found that activation in the hippocampus predicted subsequent increases in ventral anterior cingulate cortex (vACC) activity in depression, and that activity in the medial prefrontal cortex and vACC were mutually reinforcing in MDD. Their findings advance the neural theory of depression by detailing specific patterns of limbic excitation in MDD, by making explicit the primary role of limbic inhibition of the dorsal cortex in the cortico-limbic relation posited to underlie depression, and by presenting an integrated neurofunctional account of altered dopamine function in this disorder.

\section{Prevention and treatment}

Finally, the most clinically useful implications of research on a given disease are prevention and treatment. Two of the articles in this issue cover these areas. Regarding prevention, in an exemplary translational paper, Chen et $a l^{9}$ from Vancouver, Canada, show that in humans, maternal warmth buffers the effects of low early-life socioeconomic status (SES) on pro-inflammatory signaling in adulthood. The authors' hypothesis was that adults who grew up in low SES households but who experienced high levels of maternal warmth would be protected from the pro-inflammatory states typically associated with low SES. They convincingly showed that low early-life SES individuals who had mothers who expressed high warmth toward them, exhibited less Toll-like receptor-stimulated production of interleukin 6, and reduced bioinformatic indications of proinflammatory transcription factor activity $(\mathrm{NF}-\kappa \mathrm{B})$ and immune-activating transcription factor activity (AP-1) compared with those who were low in SES early in life but experienced low maternal warmth. This indicates that positive environmental interventions early in life may have long-lasting impact.

Treatment mechanisms have been insightfully addressed by Anacker et al. ${ }^{10}$ from London, who present in this issue's cover article exciting data on a mechanism of action for antidepressant drugs. In a paper entitled 'Antidepressants increase human hippocampal neurogenesis by activating the glucocorticoid receptor,' they demonstrate that treatment (for 3-10 days) with the antidepressant sertraline increased neuronal differentiation via a glucocorticoid receptor-dependent mechanism. Their experiments point toward a critical role for glucocorticoid receptors in antidepressant-induced modulation of neurogenesis in humans.

These eight papers exemplify major advances being made in depression research in 2011. Such progress provides ample evidence that we are on the right track towards generating the scientific foundation and the evidence needed to decrease the burden of this common and complex disease.

J Licinio and M-L Wong
John Curtin School of Medical Research,
The Australian National University,
Canberra, ACT, Australia
E-mail: julio.licinio@anu.edu.au
or mali.wong@anu.edu.au

\section{References}

1 Milane MS et al. PLoS Med 2006; 3: e190.

2 Fox M. Reuters 4 Aug, 2009 Report; http://www.reuters.com/ article/2009/08/04/usantidepressants-usa-idUSTRE5725E720090804.

3 Demirkan A et al. Mol Psychiatry 2011; 16: 773-783.

4 Peñas-LLedó EM et al. Mol Psychiatry 2011; 16: 691-692.

5 van Donkelaar EL et al. Mol Psychiatry 2011; 16: 695-713.

6 Regev L et al. Mol Psychiatry 2011; 16: 714-728.

7 Shelton RC et al. Mol Psychiatry 2011; 16: 751-762.

8 Hamilton JP et al. Mol Psychiatry 2011; 16: 763-772.

9 Chen E et al. Mol Psychiatry 2011; 16: 729-737.

10 Anacker C et al. Mol Psychiatry 2011; 16: 738-750. 